\title{
Reduced expression of caspase-8 and cleaved caspase-3 in pancreatic ductal adenocarcinoma cells
}

\author{
KATARZYNA JAKUBOWSKA ${ }^{1}$, KATARZYNA GUZIŃSKA-USTYMOWICZ ${ }^{1}$, WALDEMAR FAMULSKI ${ }^{2}$, \\ DARIUSZ CEPOWICZ ${ }^{3}$, DOROTA JAGODZINSSKA ${ }^{1}$ and ANNA PRYCZYNICZ ${ }^{1}$
}

\author{
Departments of ${ }^{1}$ General Pathomorphology, ${ }^{2}$ Medical Pathomorphology and ${ }^{3}$ General Surgery and Gastroenterology, \\ Medical University of Białystok, Białystok 15-276, Poland
}

Received December 9, 2014; Accepted November 24, 2015

DOI: $10.3892 / 01.2016 .4125$

\begin{abstract}
Pancreatic ductal adenocarcinoma (PDAC) is a rare neoplasm that affects the gastrointestinal system, and is characterized by a high mortality rate. It has been demonstrated that apoptosis has a significant role in the regulation of cancer cells. Therefore, the aim of the present study was to immunohistochemically assess the expression of proteins belonging to the caspase family, namely caspase- 8 , pro-caspase- 3 and cleaved (active) caspase- 3 in pancreatic cancer. The study group consisted of 29 patients exhibiting PDAC. Protein expression was evaluated by immunohistochemical methods. The expression of caspase- 8 in normal cells was negative in $17.2 \%$ of cases and positive in $82.8 \%$ of cases. All cases demonstrated pro-caspase-3 expression in normal pancreatic cells, compared with $93.1 \%$ of cancer cells. Staining for activated caspase-3 was positive in 27 normal tissue cases, compared with positivity in only 10 cancer cases. Caspase- 8 expression positively correlated with cleaved caspase- 3 expression in the cytoplasm of cancer cells $(\mathrm{P}<0.002)$. Caspase-3 expression was identified to correlate with inflammatory peritumoral infiltration $(\mathrm{P}<0.015)$. No correlation was observed between caspase expression and any other clinicopathological parameters. The results of the present study demonstrated aberrant initiation of cancer cell apoptosis in PDAC via a decrease in caspase- 8 expression, which may lead to disorders in the activation of effector caspase-3.
\end{abstract}

\section{Introduction}

Pancreatic cancer is a malignancy with a particularly poor prognosis (1). Pancreatic ductal adenocarcinoma (PDAC) accounts for $85 \%$ of all cases of pancreatic cancer, and is

Correspondence to: Mrs. Katarzyna Jakubowska, Department of General Pathomorphology, Medical University of Białystok, 24a M. Skłodowskiej-Curie Street, Białystok 15-276, Poland E-mail:kathian@wp.pl

Key words: pancreatic ductal cancer, apoptosis, caspase expression, immunohistochemistry the most frequently observed type of malignancy derived primarily from pancreatic cells (2). It is equally common in men and women, and mainly involves the head of the pancreas (2). In the majority of cases, the tumor already infiltrates the adjacent structures at the time of diagnosis, including the common bile duct, duodenum and ampulla (1). Lesions are typically irreversible, and the 5-year survival rate is $1-2 \%$ (1). The low percentage of survival in pancreatic cancer cases results principally from the weak response of cancer cells to treatment, which is mainly due to their high level of resistance to apoptotic stimuli (1).

Apoptosis, also known as programmed cell death, is involved in the physiological maintenance of normal cell homeostasis, as well as having a role in pathological lesions that accompany the course of various diseases $(3,4)$. The caspase activation mechanism is a major signaling pathway leading to apoptosis (1). Caspases constitute a group of cysteine proteases that occur in an inactive and an activated form (4). They become activated via the action of proteases, including their mutual activation. Caspases may be divided into effectors (caspase-3, -6 and -7) and initiators (caspase-8 and -9) (4). Two mechanisms that lead to the activation of the caspase initiators have been proposed: i) A mechanism by which the inactive form of caspase- 8 is activated upon binding of its death receptor to a protein containing a death domain, and whereby the activated protein directly stimulates effector caspases such as caspase-3 $(1,3,4)$; ii) and an alternative B-cell lymphoma 2 (Bcl-2) family-dependent mechanism that involves the cytosol and is associated with the permeabilization of the mitochondrial outer membrane and subsequent release of cytochrome $c$, apoptotic protease activating factor 1 and additional apoptotic agents. This leads to the formation of apoptosomes that activate caspase-9, which subsequently triggers a cascade of effector caspases (caspase-3 and -7) that induce the process of apoptosis (5). Disorders in these signaling pathways are associated with cancer cell immortality (5).

Therefore, the aim of the present study was the immunohistochemical assessment of the expression of caspase- 8 and the inactive and cleaved (active) forms of caspase- 3 in the tissues of patients exhibiting PDAC, and the subsequent analysis of the association of caspase expression in these patients with specific clinicopathological parameters. 

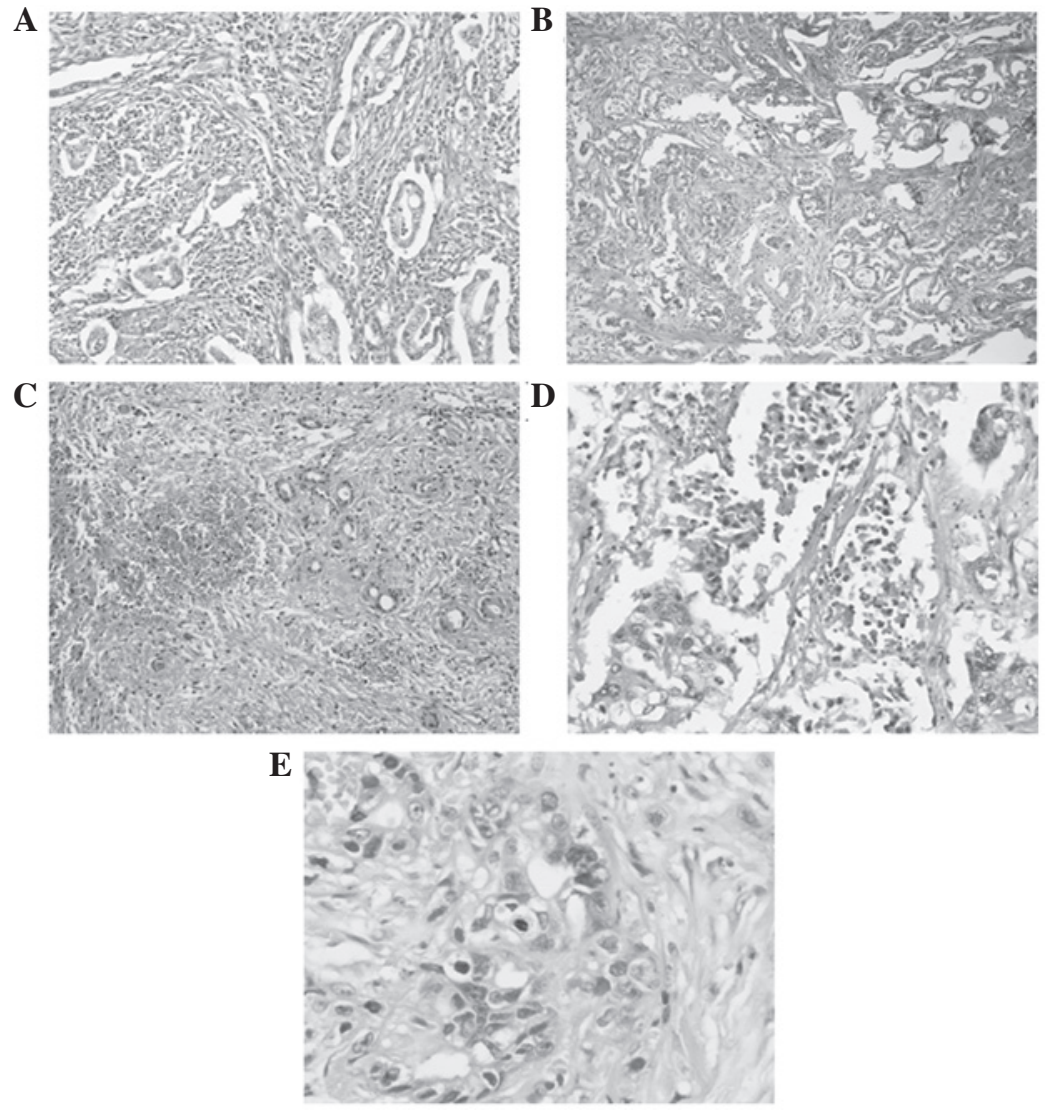

Figure 1. Histopathological characteristics of pancreatic cancer tissue. (A) A moderate grade of intratumoral inflammation was observed in the stroma. Magnification, x20. (B) A high grade of desmoplasia (magnification, x20), (C) single focus of hemorrhage (magnification, x20), (D) weak or moderate tumor necrosis (magnification, $\mathrm{x} 40$ ), (E) low mitotic index and apoptotic bodies (magnification, $\mathrm{x} 40$ ) were observed in the majority of cases. Hematoxylin and eosin staining.

\section{Materials and methods}

Patients and tumors. The present study group comprised 29 patients exhibiting PDAC, who underwent surgery at the Department of General Surgery and Gastroenterology at the Medical University of Białystok (Białystok, Poland). The present study included 6 female and 23 male patients, whose ages ranged from 41 to 75 years. Sections $(4-\mu \mathrm{m})$ were cut from paraffin blocks and stained using hematoxylin and eosin. Routine histopathological assessment of the sections referred to the histological type, malignancy grade $(\mathrm{G})$, clinicopathological TNM status, regional lymph node involvement and presence of distant metastases. Pancreatic adenocarcinomas were moderately differentiated (G2) in $25 / 29$ cases and poorly differentiated (G3) in 4/29 cases. Adenocarcinomas were classified as mucinous in 3/29 cases and non-mucinous in 26/29 cases. Lymph node involvement was noted in 12/29 cases and metastasis to distant organs (including liver and intestine) was observed in 9/29 cases. In addition, the degrees of inflammation, desmoplasia, necrosis and foci of hemorrhage were assessed. A weak inflammatory response was observed in 9 cases, a moderate response in 10 cases and a strong response in 10 cases. Numerous foci of hemorrhage were observed in 5 cases, a single focus of hemorrhage was observed in 10 cases and foci of hemorrhage were absent in 14 cases. Pancreatic adenocarcinoma was associated with weak or moderate necrosis in 14 cases. Low- and high-grade desmoplastic tumors were noted in 12 and 17 cases, respectively. The histological features of pancreatic adenocarcinoma are presented in Fig. 1.

The present study was performed in accordance with the Declaration of Helsinki for human experimentation and received approval from the local bioethics committee of the Medical University of Białystok.

Mitotic index (MI) and apoptotic bodies examination. MI was counted as the number of mitotic figures/10 high-power fields (magnification, $\mathrm{x} 40$ ), and samples were categorized into two groups with cut-off values of $<5$ and $>5$ MI. Apoptotic bodies were identified as fragmented nuclear debris with cytoplasmic vacuoles, and counted as described above for MI. Samples were classified into two groups with cut-off values of $<5$ and $>5$ apoptotic bodies.

Immunohistochemical analysis. Formalin-fixed and paraffin-embedded tissue specimens were cut on a microtome into $4-\mu \mathrm{m}$ sections, which were subsequently deparaffinized with xylene (Chempur, Piekary Śląskie, Poland) and hydrated in graded alcohol (Chempur). In order to visualize the antigens of pro-caspase- 3 and caspase- 8 , the sections were heated in a microwave oven (LAZNIE-WLS-084; Adverti, Lodz, Poland) for $20 \mathrm{~min}$ in citrate buffer (pH 6.0; Sigma-Aldrich, St. Louis, MO, USA). The antigen of cleaved caspase- 3 was visualized by heating the sections for $20 \mathrm{~min}$ in ethylenediaminetetraacetic acid buffer (pH 9.0; Sigma-Aldrich). Sections were incubated 
Table I. Immunohistochemical characteristics of caspase expression in normal and pancreatic tumor cells.

\begin{tabular}{|c|c|c|c|c|}
\hline \multirow[b]{2}{*}{ Protein expression } & \multicolumn{2}{|c|}{ Normal ductal cells, $\mathrm{n}(\%)$} & \multicolumn{2}{|c|}{ Pancreatic ductal cancer cells, $\mathrm{n}(\%)$} \\
\hline & Negative & Positive & Negative & Positive \\
\hline Caspase-8 & $5(17.2)$ & $24(82.8)$ & $14(48.3)$ & $15(51.7)$ \\
\hline Pro-caspase-3 & $0(0.0)$ & $29(100.0)$ & $2(6.9)$ & $27(93.1)$ \\
\hline Active caspase- 3 & $2(6.9)$ & $27(93.1)$ & $19(65.5)$ & $10(34.5)$ \\
\hline
\end{tabular}

with $3 \%$ hydrogen peroxide solution (Chempur) for $20 \mathrm{~min}$ in order to block endogenous peroxidase activity. Subsequently, incubation was performed with rabbit monoclonal antibody against human pro-caspase-3 (cat. no. ab32150; Abcam, Cambridge, UK; incubated for $1 \mathrm{~h}$, dilution, 1:500), rabbit polyclonal immunoglobulin $\mathrm{G}$ against human/mouse cleaved caspase-3 (cat. no. AF835; R\&D Systems, Inc., Minneapolis, MN, USA; incubated for $30 \mathrm{~min}$, dilution, 1:400) and rabbit monoclonal antibody against human caspase- 8 (cat. no. sc-56070; Santa Cruz Biotechnology, Inc., Dallas, TX, USA; incubated for $1 \mathrm{~h}$; dilution, 1:60). The reaction was performed using Novostain Super ABC Kit (universal) (Leica Microsystems, Inc.) at room temperature. A color reaction for peroxidase was developed with DAB Chromogen Solution (Dako, Glostrup, Denmark). Negative control sections were incubated without primary antibody in citrate buffer. All sections were counterstained with hematoxylin.

Immunohistochemical staining was evaluated by two independent pathologists who were blinded to the clinical information. The expression of the investigated proteins was observed in the cytoplasm of normal ductal epithelial and tumor cells in all the cases analyzed. Caspase-8, pro-caspase-3 and cleaved caspase-3 expression was determined using a semiquantitative method, and was defined in relation to the intensity of staining ( 0 , absent; 1 , weak; 2 , moderate; and 3 , strong) and the percentage of positive tumor cells. H-score was derived by adding the percentages of stained cells at each intensity and multiplying by the weighted intensity of staining, according to the following formula: $\mathrm{H}$-score $=(\%$ unstained cells $\times 0)+(\%$ weakly stained cells x 1$)+(\%$ moderately stained cells x 2) $+(\%$ strongly stained cells x 3$)$. Scores ranged from 0 to 300. The study group was divided into negative $(\mathrm{H}$-score $<150)$ and positive cases $(\mathrm{H}$-score $>150)$.

Statistical analysis. Statistical analysis was performed using STATISTICA version 10.0 software (StatSoft, Inc., Tulsa, OK, USA). Correlations between the clinicopathological parameters and the expression levels of caspases exhibited by the patients were calculated using the Spearman's rank correlation test. $\mathrm{P}<0.05$ was considered to indicate a statistically significant difference. Missing data were removed in pairs when one of the clinicopathological parameters were unavailable.

\section{Results}

Expression of caspase-8, pro-caspase-3 and cleaved caspase-3 is observed in normal pancreas and cancerous cells. Color reaction corresponding to positive protein expression of caspase- 8 , pro-caspase- 3 and active caspase- 3 was detected in the cytoplasm of normal pancreatic cells (including ductal epithelial, lobular and single pancreatic cells of the islets of Langerhans) and in PDAC cells. Due to the origin of PDAC, only the normal glandular epithelium of the ducts was precisely analyzed and used in the statistical analysis. Furthermore, no expression of the investigated proteins was identified in normal pancreatic stroma.

The expression of caspase- 8 in normal cells was negative in $17.2 \%$ and positive in $82.8 \%$ of cases. However, PDAC cells demonstrated positivity for caspase- 8 expression in $\sim 50 \%$ of cases. All cases demonstrated pro-caspase-3 expression in normal pancreatic cells, compared with $93.1 \%$ of cancer cells. Activated caspase-3 was expressed in normal tissue in 27 cases, compared with only 10 cases in PDAC cells. A substantial decrease was observed in the expression of caspase- 8 and active caspase- 3 in cancer cells, compared with the high expression levels of these proteins in normal pancreatic cells. The expression of caspase-8, pro-caspase-3 and active caspase- 3 in normal and cancerous cells is summarized in Table I and Fig. 2.

A correlation exists between the protein expression levels of the investigated caspases and certain clinicopathological parameters. Statistical analysis revealed a strong positive correlation between the expression of caspase- 8 and active caspase- 3 in cancer cells $(\mathrm{P}<0.002)$. A reduction in caspase- 8 expression was accompanied by a decrease in the levels of active caspase-3. Expression of caspase-3 was identified to correlate with inflammatory peritumoral infiltration $(\mathrm{P}<0.015)$. No correlation was observed between the expression levels of the investigated proteins and any other clinicopathological parameters of the patients, including gender, age, type of cancer, malignancy grade, presence of metastasis to lymph nodes or distant organs, grade of desmoplasia, foci of hemorrhage, necrosis, MI and apoptotic bodies (Table II).

\section{Discussion}

Normal pancreatic cells, identical to other body cells, undergo the physiological process of controlled cell death, known as apoptosis (6). This mechanism conditions normal cell function and enables maintenance of homeostasis (7). The process of apoptosis involves the Bcl-2 family-dependent signaling pathway and the caspase-regulated signaling pathway (7). It has been previously demonstrated that the action of these proteins is disrupted in pancreatic cells affected by various pathologies (6-8). 

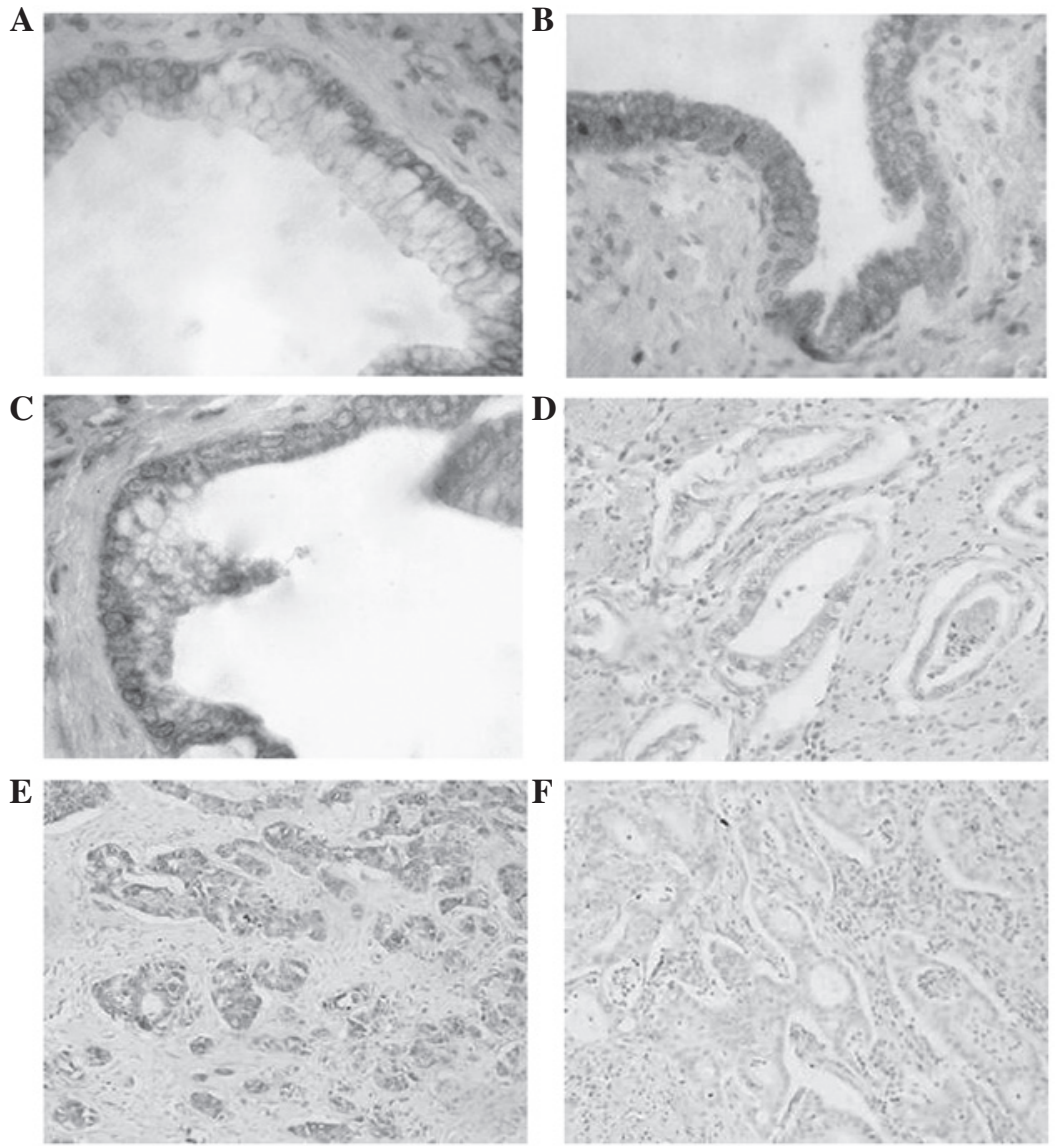

Figure 2. Expression of caspase-8, pro-caspase-3 and active caspase-3 in normal and cancerous pancreatic ductal cells. (A) Positive expression of caspase- 8 in ductal cells was observed in the majority of cases. (B) Positive expression of pro-caspase-3 was observed in normal tissues. (C) Strong expression of cleaved caspase-3 was observed in normal cells. (D) Caspase-8 expression was negative in $\sim 50 \%$ of cancer cell cases. (E) Positive expression of pro-caspase-3 was observed in cancerous tissues. (F) A lack of expression of cleaved caspase-3 was observed in pancreatic ductal adenocarcinoma. Immunohistochemical staining. Magnification, x60 (A-C) and x40 (D-F).

In normal pancreatic tissue, positive expression of caspase- 8 has been identified in the cytoplasm of glandular cells, single islets of Langerhans and ductal epithelial cells (8). The results of the present study are consistent with the observations reported by Virkajärvi et al (9), who described the expression of caspase-8 in all types of structural cells in the pancreas, as well as strong expression of caspase- 8 in inflamed areas surrounding the islets of Langerhans. Analysis of caspase- 8 expression in cancer cells confirmed its presence in $74 \%$ of patients exhibiting pancreatic adenocarcinoma, and strong expression was observed in a small percentage of cases (9). In the present study, $93 \%$ of cases exhibiting PDAC demonstrated positive expression of caspase-8. Similar results have been reported in other gastrointestinal tumors, including gastric or colorectal cancer. Sträter et al (10) reported moderate or strong caspase- 8 expression in the cancer cells of a small number of colon tumors. Shintani et al (11) observed immunopositive cells for caspase- 8 in $\sim 50 \%$ of gastric and colorectal adenocarcinoma cases. Previous studies have indicated that this may be due to disturbances in apoptosis induction within the Fas/Fas ligand system, or alternative death ligands, including tumor necrosis factor (TNF) or TNF-related apoptosis-inducing ligand, which may cause dysfunction in initiator caspases such as caspase-8 and inhibition of apoptosis during its initial stage $(12,13)$.
The present study additionally focused on the expression of caspase- 3 in its precursor and activated forms in normal pancreatic and cancer cells. A positive pro-caspase-3 color reaction was identified by immunohistochemistry in the cytoplasm of normal and cancerous cells in the majority of cases analyzed. Thus, it was speculated that PDAC cells may retain the potential to produce the precursor form of caspase- 3 . To the best of our knowledge, the present study is the first to assess the expression of the two forms of caspase-3 separately. Virkajärvi et al (9) evaluated the expression of the precursor and active forms of caspase- 3 using one antibody. Expression of caspase- 3 was observed in $80 \%$ of patients exhibiting pancreatic adenocarcinoma. However, no correlation was identified between apoptotic index and caspase- 3 expression, which may indicate the expression of the inactive form of caspase- 3 in the cancer cells of these patients (9). Satoh et al (14) observed high expression levels of caspase-3 in PDAC cells in the majority of cases evaluated, and suggested that the precursor form of caspase-3 may have contributed to the color observed upon immunohistochemical staining (14). In that study, caspase-3 expression was assessed in the cytoplasm and nuclei of PDAC cells, and a correlation was observed between cytoplasmic expression of caspase- 3 and malignant tumor stage. In addition, an association was observed between nuclear reaction and benign tumor phenotype (14). 
Table II. Clinicopathological characteristics of patients exhibiting pancreatic ductal adenocarcinoma.

\begin{tabular}{|c|c|c|c|c|c|c|c|}
\hline \multirow[b]{2}{*}{ Parameter } & \multirow[b]{2}{*}{ Patients, n (\%) } & \multicolumn{2}{|c|}{ Caspase- 8} & \multicolumn{2}{|c|}{ Pro-caspase-3 } & \multicolumn{2}{|c|}{ Active caspase-3 } \\
\hline & & $\mathrm{R}$ & $\mathrm{P}$-value & $\mathrm{R}$ & $\mathrm{P}$-value & $\mathrm{R}$ & P-value \\
\hline \multicolumn{8}{|l|}{ Age, years } \\
\hline$\leq 60$ & $14(48.3)$ & & & & & & \\
\hline$>60$ & $15(51.7)$ & -0.017 & 0.890 & -0.165 & 0.678 & 0.188 & 0.342 \\
\hline \multicolumn{8}{|l|}{ Gender } \\
\hline Male & $23(79.3)$ & & & & & & \\
\hline Female & $6(20.7)$ & 0.215 & 0.574 & -0.016 & 0.076 & 0.199 & 0.886 \\
\hline \multicolumn{8}{|c|}{ Adenocarcinoma type } \\
\hline Non-mucinous & $26(89.7)$ & & & & & & \\
\hline Mucinous & $3(10.3)$ & 0.193 & 0.295 & 0.135 & 0.512 & -0.079 & 0.637 \\
\hline \multicolumn{8}{|c|}{ Grade of malignancy } \\
\hline G2 & $25(86.2)$ & & & & & & \\
\hline G3 & $4(13.8)$ & -0.013 & 0.985 & 0.159 & 0.439 & -0.095 & 0.578 \\
\hline \multicolumn{8}{|c|}{ Lymph node metastasis } \\
\hline Absent & $17(58.6)$ & & & & & & \\
\hline Present & $12(41.4)$ & 0.036 & 0.873 & 0.079 & 0.701 & 0.008 & 0.863 \\
\hline \multicolumn{8}{|l|}{ Distant metastasis } \\
\hline Absent & $20(69.0)$ & & & & & & \\
\hline Present & $9(31.0)$ & -0.083 & 0.661 & 0.023 & 0.911 & -0.146 & 0.389 \\
\hline \multicolumn{8}{|c|}{ Inflammation in peritumoral stroma } \\
\hline Absent & $2(6.9)$ & & & & & & \\
\hline Weak & $9(31.0)$ & & & & & & \\
\hline Moderate & $10(34.5)$ & & & & & & \\
\hline Strong & $8(27.6)$ & 0.320 & 0.082 & 0.143 & 0.195 & 0.404 & $<0.015$ \\
\hline \multicolumn{8}{|c|}{ Grade of desmoplasia } \\
\hline Low & $12(41.4)$ & & & & & & \\
\hline High & $17(58.6)$ & -0.242 & 0.193 & -0.128 & 0.679 & -0.316 & 0.068 \\
\hline \multicolumn{8}{|l|}{ Foci of hemorrhage } \\
\hline Absent & $14(48.3)$ & & & & & & \\
\hline Single & $10(34.5)$ & & & & & & \\
\hline Numerous & $5(17.2)$ & 0.134 & 0.487 & 0.032 & 0.868 & 0.008 & 0.964 \\
\hline \multicolumn{8}{|l|}{ Necrosis } \\
\hline Absent & $16(55.2)$ & & & & & & \\
\hline Weak & $7(24.1)$ & & & & & & \\
\hline Moderate & $6(20.7)$ & & & & & & \\
\hline Strong & $0(0.0)$ & -0.040 & 0.837 & -0.294 & 0.121 & 0.089 & 0.643 \\
\hline \multicolumn{8}{|l|}{ Mitotic index } \\
\hline$<5$ & $27(93.1)$ & & & & & & \\
\hline$>5$ & $2(6.9)$ & 0.248 & 0.735 & -0.087 & 0.970 & 0.130 & 0.873 \\
\hline \multicolumn{8}{|l|}{ Apoptotic bodies } \\
\hline$<5$ & $25(86.2)$ & & & & & & \\
\hline$>5$ & $4(13.8)$ & 0.146 & 0.267 & -0.093 & 0.927 & -0.034 & 0.055 \\
\hline \multicolumn{8}{|c|}{ Protein expression in cancer cells } \\
\hline Caspase- 8 & $27(93.1)$ &.- & - & 0.195 & 0.308 & 0.542 & $<0.002$ \\
\hline Pro-caspase-3 & $26(86.7)$ & 0.195 & 0.308 &.- & - & -0.212 & 0.269 \\
\hline Active caspase-3 & $21(72.4)$ & 0.542 & $<0.002$ & -0.212 & 0.269 &.- & - \\
\hline
\end{tabular}

R, Spearman's Rank correlation coefficient; -, not available. 
The present study particularly focused on the expression of activated caspase-3. More frequent expression of cleaved caspase-3 was observed in normal pancreatic cells, compared with pancreatic duct cell tumors. In the present study, a statistically significant correlation was identified between caspase- 8 and active caspase- 3 expression in the tumor mass. A decrease in the expression levels of caspase- 8 or -3 caused a reduction in the expression levels of the other, which confirmed the involvement of these two proteins in the common apoptotic signaling pathway of PDAC cells. Meggiato et al (15) noted an increased percentage of positive expression of cleaved caspase-3 in the cytoplasm of tumor cells, compared with the cytoplasm of epithelial cells that lined the pancreatic ducts. It was suggested that the strong expression of active caspase-3 in pancreatic tumor cells, as opposed to normal ductal cells, indicated stimulation of the apoptosis-inducing mechanisms (15). Similarly, Luo et al (16) reported an increase in the expression of cleaved caspase-3 in PDAC tissue (43.9\%), compared with normal tissue $(3.5 \%)$. Furthermore, statistically significant correlations were noted between caspase-3 expression and high tumor grade, tumor location in the head of the pancreas, cyclin D1 gene amplification and presence of polysomy of chromosome 11 (16). A detailed analysis of these patients additionally revealed an association between positive expression of active caspase-3 and shorter survival time in patients exhibiting PDAC, and the expression of active caspase- 3 was identified as an independent risk factor (16). In contrast to PDAC, high expression levels of cleaved caspase- 3 have been associated with positive prognosis in patients diagnosed with colorectal cancer (17). The previously mentioned reports are not consistent with the results of the present study, which may be due to the small size of the current patient cohort. However, reduced expression of effector cleaved caspase-3 may be associated with a lack of adequate agents to trigger the activation of the protein, including caspase-8, or may result from disorders in the Bcl-2 family-dependent signaling pathway of apoptosis, which conditions the levels of cytochrome $c$ present in the cytosol (18). In contrast with normal cells, pancreatic cancer cells demonstrate a reduced ability to activate caspases via cytochrome $c$ (18). It has been suggested that cancer cells may require increased levels of cytochrome $c$ in order to activate the apoptotic signaling pathway of caspases (1). The reduced capacity of caspase- 3 stimulation in PDAC cells may induce their resistance to apoptosis, as well as determining their longevity and invasiveness (18). In consequence, PDAC belongs to a group of tumors characterized by short survival times and poor prognoses (18).

Despite the small size of the patient cohort in the current study, the findings of the present study suggest that pancreatic cancer may involve disturbances to the initiation of cancer cell apoptosis via a decrease in the expression of caspase-8, which may cause dysregulation in the activation of effector caspase-3. Therefore, assessment of the expression of caspase- 8 and cleaved caspase- 3 in the tissues of patients exhibiting PDAC may aid the determination of the degree of apoptotic activity in pancreatic cancer cells. This may be utilized in the future to develop targeted therapies for the treatment of PDA using activators of the proteins investigated in the present study.

\section{References}

1. Gukovskaya AS and Pandol SJ: Cell death pathways in pancreatitis and pancreatic cancer. Pancreatology 4: 567-586, 2004.

2. Reid MD, Bagci P and Adsay NV: Histopathologic assessment of pancreatic cancer: Does one size fit all? J Surg Oncol 107: 67-77, 2013.

3. Adams JM: Ways of dying: Multiple pathways to apoptosis. Genes Dev 17: 2481-2495, 2003.

4. Schultz DR and Harrington WJ Jr: Apoptosis: Programmed cell death at a molecular level. Semin Arthritis Rheum 32: 345-369, 2003.

5. Li P, Nijhawan D, Budihardjo I, Srinivasula SM, Ahmad M, Alnemri ES and Wang X: Cytochrome $c$ and dATP-dependent formation of Apaf-1/caspase-9 complex initiates an apoptotic protease cascade. Cell 91: 479-489, 1997.

6. Steer ML: Early events in acute pancreatitis. Baillieres Best Pract Res Clin Gastroenterol 13: 213-225, 1999.

7. Tomita T: Cleaved caspase-3 immunocytochemical staining for pancreatic islets and pancreatic endocrine tumors: A potential marker for biological malignancy. Islets 2: 82-88, 2010.

8. Butler AE, Janson J, Bonner-Weir S, Ritzel R, Rizza RA and Butler PC: Beta-cell deficit and increased beta-cell apoptosis in humans with type 2 diabetes. Diabetes 52: 102-110, 2003.

9. Virkajärvi N, Pääkkö P and Soini Y: Apoptotic index and apoptosis influencing proteins bcl-2, mcl-1, bax and caspases 3,6 and 8 in pancreatic carcinoma. Histopathology 33: 432-439, 1998.

10. Sträter J, Herter I, Merkel G, Hinz U, Weitz J and Möller P: Expression and prognostic significance of APAF-1, caspase- 8 and caspase-9 in stage II/III colon carcinoma: Caspase-8 and caspase-9 is associated with poor prognosis. Int J Cancer 127: 873-880, 2010.

11. Shintani M, Sangawa A, Yamao N, Miyake T and Kamoshida S: Immunohistochemical analysis of cell death pathways in gastrointestinal adenocarcinoma. Biomed Res 32: 379-386, 2011.

12. Kornmann M, Ishiwata T, Kleeff J, Beger HG and Korc M: Fas and Fas-ligand expression in human pancreatic cancer. Ann Surg 231: 368-379, 2000.

13. Tsuji S, Hosotani R, Yonehara S, Masui T, Tulachan SS, Nakajima S, Kobayashi H, Koizumi M, Toyoda E, Ito D, et al: Endogenous decoy receptor 3 blocks the growth inhibition signals mediated by Fas ligand in human pancreatic adenocarcinoma. Int J Cancer 106: 17-25, 2003.

14. Satoh K, Kaneko K, Hirota M, Toyota T and Shimosegawa T: The pattern of CPP32/caspase-3 expression reflects the biological behavior of the human pancreatic duct cell tumors. Pancreas 21: 352-357, 2000.

15. Meggiato T, Calabrese F, De Cesare CM, Baliello E, Valente M and Del Favero G: C-JUN and CPP32 (CASPASE 3) in human pancreatic cancer: Relation to cell proliferation and death. Pancreas 26: 65-70. 2003.

16. Luo Y, Qiu Z, Tian L, Zhu G, Feng Y, Yi M, Chen X, Wang L, $\mathrm{Li} \mathrm{C}$ and Huang Q: Identification of novel predictive markers for the prognosis of pancreatic ductal adenocarcinoma. Hum Pathol 44: 69-76, 2013.

17. Noble P, Vyas M, Al-Attar A, Durrant S, Scholefield J and Durrant L: High levels of cleaved caspase- 3 in colorectal tumour stroma predict good survival. Br J Cancer 108: 2097-2105, 2013.

18. Vaquero EC, Edderkaoui M, Nam KJ, Gukovsky I, Pandol SJ and Gukovskaya AS: Extracellular matrix proteins protect pancreatic cancer cells from death via mitochondrial and nonmitochondrial pathways. Gastroenterology 125: 1188-1202, 2003. 